\title{
Optimizing the current ramp-up phase for the hybrid ITER scenario
}

\author{
G M D Hogeweij ${ }^{1}$, J.-F. Artaud ${ }^{2}$, T.A. Casper ${ }^{3}$, J. Citrin ${ }^{1}$, \\ F. Imbeaux ${ }^{2}$, F. Köchl ${ }^{4}$, X. Litaudon ${ }^{2}$, I. Voitsekhovitch ${ }^{5}$, and \\ the ITM-TF ITER Scenario Modelling group \\ ${ }^{1}$ FOM Institute DIFFER - Dutch Institute for Fundamental Energy Research, \\ Association EURATOM-FOM, Trilateral Euregio Cluster, P.O.Box 1207, Nieuwegein, \\ The Netherlands, www.differ.nl \\ ${ }^{2}$ CEA, IRFM, F-13108 Saint Paul lez Durance, France \\ ${ }^{3}$ ITER Organization, F-13115 Saint Paul lez Durance, France \\ ${ }^{4}$ Association EURATOM-ÖAW/ATI, Atominstitut, TU Wien, 1020 Vienna, Austria \\ ${ }^{5}$ EURATOM/CCFE Fusion Association, Culham Science Centre, Abingdon OX14 \\ 3DB, UK
}

\begin{abstract}
The current ramp-up phase for the ITER hybrid scenario is analyzed with the CRONOS integrated modelling suite. The simulations presented in this paper show that the heating systems available at ITER allow, within the operational limits, the attainment of a hybrid $q$ profile at the end of the current ramp-up. A reference ramp-up scenario is reached by a combination of NBI, ECCD (UPL) and LHCD. A heating scheme with only NBI and ECCD can also reach the target q profile; however, LHCD can play a crucial role in reducing the flux consumption during the ramp-up phase. The optimum heating scheme depends on the chosen transport model, and on assumptions on parameters like $n_{\mathrm{e}}$ peaking, edge $T_{\mathrm{e}, \mathrm{i}}$ and $Z_{\mathrm{eff}}$. The sensitivity of the current diffusion on parameters that are not easily controlled, shows that development of real time control is important to reach the target $q$ profile. A first step in that direction has been indicated in this paper. Minimizing resistive flux consumption and optimizing the $q$ profile turn out to be conflicting requirements. A trade-off between these two requirements has to be made. In this paper it is shown that fast current ramp with L-mode current overshoot is at the one extreme, i.e. optimum $q$ profile at the cost of increased resistive flux consumption, whereas early H-mode transition is at the other extreme.
\end{abstract}

PACS numbers: 52.25.Fi, 52.55.Fa, 52.50.Gj

Submitted to: Nucl. Fusion 


\section{Introduction}

The scenario design of a future tokamak device like ITER naturally focuses on the main heating phase, during which fusion reactions take place. Nevertheless, the conditions to access, and eventually to terminate smoothly, the desired main heating state is also an essential topic. The main heating phase is usually carried out at high plasma current $\left(I_{\mathrm{p}}\right)$, since in a tokamak high current means high confinement. This current is ramped up from a negligible value just after the plasma breakdown to a plateau value, usually mainly by inductive means. There are several issues to be addressed during plasma current ramp phases of tokamak operation [1]. First, Magnetohydrodynamic (MHD) activity can take place and lead to early plasma termination, depending on the shape of the current density profile. Hence the design of the Poloidal Field (PF) system and plasma shape controller must allow ramping up $I_{\mathrm{p}}$ while providing stable plasma equilibrium. Secondly, a significant amount of magnetic flux is needed to ramp the plasma up inductively, thus the flux consumption during the current ramp is also a key element in the design of the PF system.

One of the operation modes foreseen for ITER is the so-called hybrid scenario [2]. Compared to the standard H-mode, this scenario is characterized by a somewhat lower $I_{\mathrm{p}}$, thus allowing a more prolonged heating phase. In this operation mode, the loss of confinement due to lower $I_{\mathrm{p}}$, is compensated by a confinement improvement brought about by a carefully tailored $q$ profile. This typical hybrid $q$ profile is characterized by $q_{\text {min }}$ near or slightly above 1 and a wide flat region [3]. Therefore, apart from the two issues mentioned above, a third issue is central to the current ramp-up phase for hybrid operation: the $q$ profile obtained at the end of the ramp-up must be optimized by applying additional heating and non-inductive current drive during the current ramp. It should be noted that experience on existing large tokamaks has shown that it is nearly impossible to restore the $q$ profile to the hybrid shape once it has relaxed to a shape with $q(0)$ clearly below 1 over a sizeable area.

This paper concentrates on the last-mentioned issue: it first reports on a systematic effort to optimize, using the available engineering knobs, the current ramp-up phase for the ITER hybrid scenario, in terms of $q$ profile attained at the end of the ramp-up phase. Then the paper addresses the sensitivity of the final $q$ profile to the assumptions made, and how to adapt the ramp-up scenario when plasma parameters deviate from assumed values. In the last part of the paper we come back briefly to the first two issues, i.e. the operational limits and the flux consumption.

There are contradictory results on the reliability of the neoclassical description of current diffusion during the current ramp-up phase. Recent work shows that in JET, using MSE measurements very early in the ramp-up, the calculated current diffusion until $q=2$ is reached is significantly faster than observed in the experiment [4]. Also in AUG the neoclassical calculation yielded a too fast current penetration during the ohmic ramp-up phase [5]. On the other hand, the calculated current diffusion in the later part of the ramp-up in JET can be matched with the observations, by adjusting 
the profiles of $Z_{\mathrm{eff}}$ and $T_{\mathrm{e}}$ within the experimental uncertainties [6]. Moreover, the observed relaxation of the $q$ profile after the $\mathrm{L}-\mathrm{H}$ transition in JET is reported to be in agreement with neoclassical calculations [7]. The emerging pattern may be that the current diffusion modelling appears to work better at low collisionality, which is good news for the ITER modelling, since the collisionality is low in ITER. In the present work we will use neoclassical resistivity for the calculation of the current density evolution. Although this may cause some overestimation of the current penetration in the early phase of the ramp-up, this systematic effect does not affect the differences between the simulations brought about by different choices of plasma and heating parameters.

Validation on the ramp-up phase of JET, AUG and Tore Supra [6, 8, 9] has shown that both empirical scaling based models and the semi-empirical Bohm/gyro-Bohm model (L-mode version, ITB shear function off, [10]) yield a good reproduction of this phase for considered discharges, in terms of $T_{\mathrm{e}}$ profile and $l_{\mathrm{i}}$. Therefore these two models have been used in the reported work. The Coppi-Tang model [11] did a good job in reproducing the current ramp-up phase in DIII-D [12], but strongly underestimated thermal transport in JET [6]; hence this model will not be used in this work.

The paper is organized as follows: first the modelling tools and ITER assumptions are discussed. Next a figure of merit for the $q$ profile is defined and the choice of the heating and current drive scheme is discussed. Then, in the central sections, the optimum ramp-up scenario (given the assumptions made) is presented, and the sensitivity of the results to several parameters is analyzed. Next we analyze whether one can gain by a faster current ramp rate or a current overshoot. Then the crucial points of operational limits and flux consumption are treated from the reconstruction of the currents in the PF coils. Finally a few words are spent on the extrapolation to the burn phase.

This work was carried out within the ITER Scenarios Modelling working group (ISM), part of the European Integrated Tokamak Modelling (ITM) Task Force.

\section{Modelling tools and assumptions}

For the simulations the CRONOS integrated suite of codes [13] was used. The core of CRONOS is a $1.5 \mathrm{D}$ transport solver, whereby $1 \mathrm{D}$ current diffusion, particle and energy equations are solved up to the separatrix, self consistently with 2D magnetic equilibrium. The magnetic equilibrium is calculated with HELENA [14]. The neoclassical transport, bootstrap current, and neoclassical resistivity are calculated with NCLASS [15]. In principle, CRONOS can model sawteeth; however, in the simulations sawteeth were not taken into account, because the goal was to stay away from sawtoothing regimes. The main simulations are done in prescribed boundary mode and are then post-processed by the FREEBIE code [16] to compute the currents in the PF coils, allowing to check that the designed scenario stays within the PF coils operational limits.

In this work, the NBI heat and current sources are calculated by NEMO/SPOT $[17,18]$, the ECRH propagation and absorption are simulated with the ray-tracing code 
REMA [19], and the LH deposition and current drive with C3PO/LUKE [20]. LHCD and ECCD have been calculated separately without accounting for possible synergy between the two. However, this synergy occurs only in very specific conditions (same location of the waves in real and velocity space); therefore it would very likely be only a small correction to the present simulations. Radial diffusion of fast electrons is not taken into account in the calculation of the driven current densities, since it has been shown to be negligible, see e.g. [21]. Other mechanisms that could lead to broadening of the LHCD and ECCD profiles, such as scattering of the waves by density fluctuations [22] were not taken into account.

As indicated in the introduction, two transport models have been used, following the outcome of the benchmark versus experiment carried out in [9]: an empirical scaling based model and the semi-empirical Bohm/gyro-Bohm model (L-mode version, ITB shear function off). For the scaling based model a fixed radial shape of the heat diffusion $\chi_{\mathrm{e}, \mathrm{i}}(\rho, t)=A(t)\left(1+6 \rho^{2}+80 \rho^{20}\right)$ is used, where $A(t)$ is adjusted at each call of the model such that the plasma thermal energy content $W_{\text {th }}$ follows a known scaling expression, e.g. the ITER-97L (L-mode) scaling [23] or the IPB98 (H-mode) scaling [24, 25]. Previous work showed that confinement during the ramp-up phase is weaker than standard Lmode [9], therefore a scaling factor smaller than 1 was needed in both cases: the optimal (and similar) agreement between experiment and simulations in the current ramp-up dataset was obtained using $L_{\mathrm{ITER} 97 \mathrm{~L}}=0.6$ and $H_{\mathrm{IPB} 98}=0.4$, respectively. Here we will use the IPB98 scaling with $H_{\mathrm{IPB} 98}=0.4$.

The ITER design and limitations are used, e.g. the designed geometries and limitations of the heating systems are used. For example, NBI is only allowed if $\left\langle n_{\mathrm{e}}\right\rangle \geq 2 \cdot 10^{19} \mathrm{~m}^{-3}$ in order to avoid shine through, and NBI can only be applied at half or full power (i.e. 16.5 or $33 \mathrm{MW}$ ). However, for numerical stability of the simulations, a finite ramp-up time of $10 \mathrm{~s}$ is assumed; this has no effect on the $q$ profiles at the end of the current ramp-up.

The following assumptions were adopted from the ITER team:

(i) An expanding ITER shape is used, starting on the LFS of the torus, with initial plasma volume $\simeq 50 \%$ of the final plasma volume. X-point formation takes place after $15 \mathrm{~s}$, when $I_{\mathrm{p}}=3.5 \mathrm{MA}$.

(ii) A flat $Z_{\text {eff }}$ profile is assumed, decreasing in time with increasing density, with an asymptotic value of 1.7 [26].

(iii) A rather low density of $n_{\mathrm{e}}=0.25 \cdot n_{\mathrm{e}}^{\mathrm{Gw}}$ is taken.

The $n_{\mathrm{e}}$ profile is assumed to be parabolic with a moderate peaking factor $n_{\mathrm{e}}(0) /\left\langle n_{\mathrm{e}}\right\rangle=1.3$. This is a compromise between the (unrealistic) flat $n_{\mathrm{e}}$ profile often used in ITER scenario predictions and the peaking factor of $\simeq 1.5$ predicted by scaling studies [27].

In addition, the simulations will be based on L-mode ramp-up. Therefore as an additional constraint is taken that the total input power should stay below the L-H threshold during the whole ramp-up phase; the L-H threshold scaling law [28] predicts for the reference density $P_{\text {LHthr }} \simeq 29 \mathrm{MW}$ at end of the current ramp-up. In section 8 
it will be argued that a transition to H-mode during the ramp-up, although profitable for the standard H-mode scenario, is not useful for the ramp-up to the hybrid scenario.

As reference scenario a moderate $I_{\mathrm{p}}$ ramp rate is chosen such that $I_{\mathrm{p}}=12 \mathrm{MA}$ is reached after $80 \mathrm{~s}$. However, also faster ramp rates and current overshoots will be considered.

Other assumptions $\left(T_{\mathrm{e}, \mathrm{i}}\right.$ (edge), initial $T_{\mathrm{e}, \mathrm{i}}$ and $\left.l_{\mathrm{i}}\right)$ are based on experimental evidence from existing large tokamaks. The initial parabolic $q$ profile is chosen by CRONOS such that it matches the given $l_{\mathrm{i}}$. Due to the high resistivity (low $T_{\mathrm{e}}$ ) in the early phase of the ramp-up, the influence of the choice of the initial $q$ profile has disappeared before halfway through the current ramp-up phase. The simulations start $1.5 \mathrm{~s}$ after breakdown, when $I_{\mathrm{p}}=0.5 \mathrm{MA}$.

\section{Figure of merit for $q$ profile}

The good performance of hybrid discharges, compared to standard H-mode, is partially due to the absence of sawteeth and other detrimental MHD modes, and partially due to a reduction of radial transport. The main driver of transport in strongly heated plasmas is thought to be the Ion Temperature Gradient (ITG) mode [29, 30]. ITG turbulence features a threshold in the inverse ion temperature gradient length, i.e. is only present when

$$
R / L_{\mathrm{T}_{\mathrm{i}}}>R / L_{\mathrm{T}_{\mathrm{i}}, \text { crit }}^{\mathrm{ITG}}
$$

where $L_{\mathrm{T}_{\mathrm{i}}}=T_{\mathrm{i}} /|\nabla(T i)|$ ITG dominated transport is then schematically given by

$$
\chi_{\mathrm{i}}=\chi_{\mathrm{i}}^{\mathrm{nc}}+\chi_{\mathrm{i}}^{\mathrm{ITG}}\left(R / L_{\mathrm{T}_{\mathrm{i}}}-R / L_{\mathrm{T}_{\mathrm{i}}, \text { crit }}^{\mathrm{ITG}}\right) \cdot H\left(R / L_{\mathrm{T}_{\mathrm{i}}}-R / L_{\mathrm{T}_{\mathrm{i}}, \text { crit }}^{\mathrm{ITT}}\right)
$$

where $H$ is the Heaviside step function. Theory predicts, and experiments confirm,

that ITG transport is stiff, i.e. $\chi_{\mathrm{i}}^{\mathrm{ITG}} \gg \chi_{\mathrm{i}}^{\mathrm{nc}}$, which implies that $R / L_{\mathrm{T}_{\mathrm{i}}}$ will never rise much above $R / L_{\mathrm{T}_{\mathrm{i}} \text {, crit. }}^{\mathrm{ITG}}$. The only exception is the core of plasmas with strong toroidal rotation, where ITG transport is much less stiff, and significant excursions of $R / L_{\mathrm{T}_{\mathrm{i}}}$ above $R / L_{\mathrm{T}_{\mathrm{i}} \text {,crit }}^{\mathrm{ITG}}$ are possible [31]. However, strong rotation cannot be achieved in ITER. Hence, the only way to improve core confinement in ITER, is to enhance $R / L_{\mathrm{T}_{\mathrm{i}}, \text { crit }}^{\mathrm{ITG}}$. Under various assumptions theories predict

$$
R / L_{\mathrm{T}_{\mathrm{i}}, \text { crit }}^{\mathrm{ITG}} \sim(1+c s / q)
$$

with $c$ a numerical constant, $c=\mathcal{O}(1)$, and where $\mathrm{s}$ is the magnetic shear $(s=r / q \nabla q)$ $[32,33]$. So one has to maximize $\langle s / q\rangle$, where the brackets denote the volume average, within the constraint for the hybrid regime that $q>1$ everywhere and that no regions with strong negative $s$ should exist. The rationale of the latter constraint is to avoid the creation of an Internal Transport Barrier (ITB), which would make control of the plasma much more complicated, and to avoid deleterious MHD, e.g. double tearing modes. We will use an empirical criterion $s>-0.5$. A high value of $\langle s / q\rangle$ is achieved by $q(0)$ close to 1 , a wide low shear region, and high $s$ in the outer part of the plasma, which are 
indeed the characteristics of a hybrid $q$ profile. For practical reasons the normalized value $\mathcal{F}=\langle s / q\rangle \cdot q_{\text {a }}$ will be used as figure of merit; its value has to be maximized at the end of the current ramp-up.

\section{Choice of heating and current drive scheme}

The most straightforward way to obtain the hybrid $q$ profile is as follows: let the discharge evolve without additional heating until $q(0)$ close to 1 , and then apply offaxis heating and CD to clamp $q(0)$ and broaden the $q$ profile. For the typical plasma conditions during the ramp-up phase, ECRH from the equatorial launcher deposits very centrally, so is unsuitable for this purpose. ICRF can, due to its wide range of possible frequencies from 40 to $70 \mathrm{MHz}$, deposit on- and off-axis; however, ICRF can only efficiently drive current when deposited on-axis [34], so is also not very useful for this purpose. The remaining heating and CD options are: NBI using the off-axis setting, i.e. with deposition radius $\rho_{\text {dep }} \sim 0.3$, LHCD (with $\rho_{d e p} \sim 0.4-0.6$ depending on plasma conditions) and the Upper Port Launcher (UPL) of ECCD. The latter has 2 antennas with different ranges of poloidal angles, with $\rho_{\text {dep }} \geq 0.4$ and 0.6 , respectively. Since ECCD and LHCD have quite narrow power deposition profiles, excessive use of one of these as only current drive source would yield a very localized net CD profile, leading to locally a strong negative shear, which should be avoided because of the risk of triggering unwanted MHD. Therefore it is better to use a combination of CD sources in such a way that the non-inductive CD is spread over a wide off-axis zone, thus compensating for the peaked ohmic drive. Figure 1 gives an example if this, taken from the reference scenario as detailed in the next section. This figure also shows that at least a combination of NBI and ECCD is needed to achieve a broad off-axis non-inductive current density profile up to $\rho \sim 0.5-0.6$; with NBI solely, still a broad $q$ profile could be achieved, but not as flat as with a combination of NBI and ECCD.

\section{Reference case}

Figure 2 shows the optimized scenario, as sketched in the previous section, for the reference case using the scaling model (left panel, full lines) Figure 3 shows the profiles of $T_{\mathrm{e}, \mathrm{i}}$ and $q$ at the end of the $I_{\mathrm{p}}$ ramp-up. For reference the figures also show the result without any additional heating. As seen from Fig.3 a good hybrid $q$ profile is reached at the end of the ramp-up. The question whether this $q$ profile can be kept during the burn phase will be treated in Section 9. We will come back to the figure of merit in the next sections.

Since the LHCD system is not foreseen in the ITER baseline design, it is important to assess the importance of LHCD for the results. Although LHCD can strongly modify the $\mathrm{q}$ profile in the early phase of the ramp-up, its effect on the q profile at the end of the ramp-up is rather modest, i.e. a scenario with LHCD replaced by extra ECCD yields a $q$ profile which is only slightly less flat, see Fig.4. However, it should be noted 


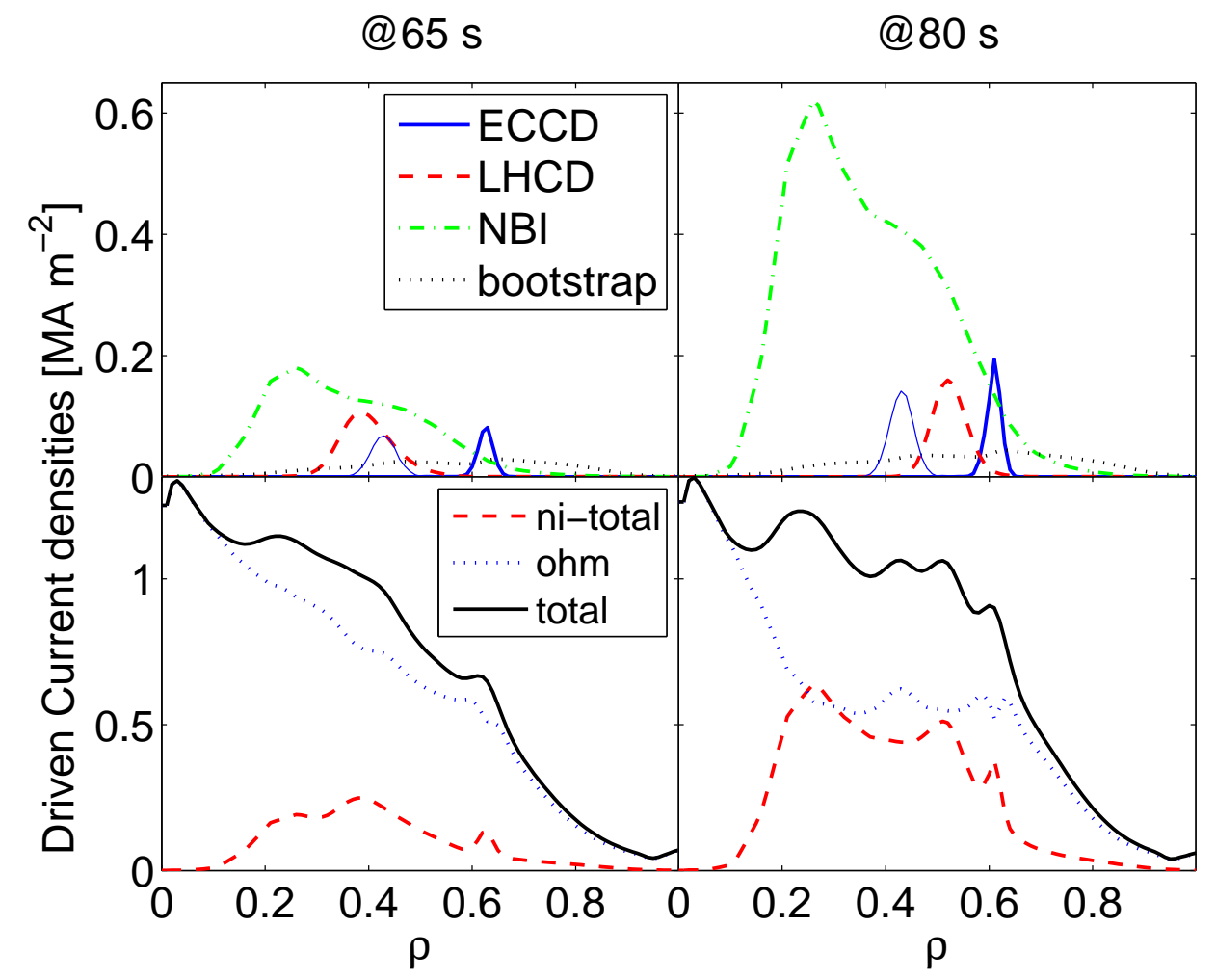

Figure 1. Driven current density profiles, plotted vs. normalized toroidal flux coordinate $\rho$ for the reference case (as described in Section 5) at $65 \mathrm{~s}$ (left) and 80 $s$ (right). The upper panels show the balanced mix of sources that is used: $8 \mathrm{MW}$ of ECCD from one of the UPL antennas (blue full), $3 \mathrm{MW}$ of $L H C D$ (red dashed) and 16.5 MW of NBI (green dashed-dotted line). Also shown is the bootstrap current density (black dotted). The lower panels show the total non-inductive driven current density (dashed red), the ohmic current density (blue dotted) and the total current density (black full line). If the total input power were allowed to exceed $P_{\mathrm{LHthr}}$, some power from the other UPL ECCD antenna could be added for an even more smooth total driven current density profile; the thin blue line in the upper panels shows the driven current density for extra $5 \mathrm{MW}$ of ECCD.

that LHCD is the most effective current drive source. Hence LHCD can play a strong role in reducing the flux consumption during the ramp-up phase [35]; a reduction of $\sim 15$ $\%$ can be reached, which would be sufficient to extend the flat top phase by hundreds of seconds.

\section{Sensitivity analysis}

Of course the optimized scheme is dependent on the chosen transport model. The Bohm/gyro-Bohm model predicts $\sim 30 \%$ lower temperatures than the scaling model, and therefore a faster current penetration; this is accounted for by switching on ECCD 


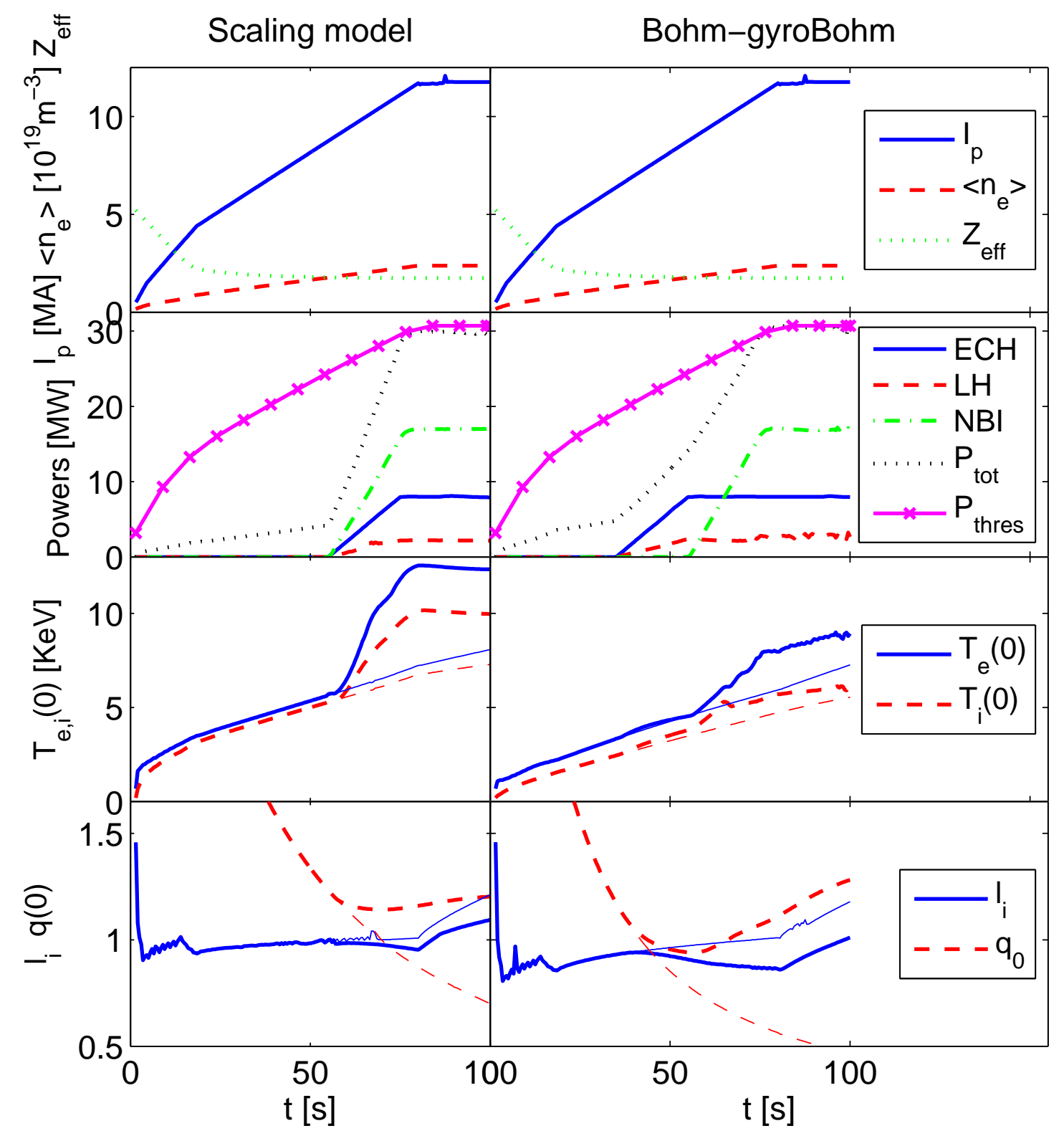

Figure 2. Time traces of the optimized scenario for the reference case, assuming scaling model (left panels) or Bohm/gyro-Bohm model (right panels). For comparison the figure also shows the time traces of $T_{\mathrm{e}, \mathrm{i}}(0), l_{\mathrm{i}}$ and $q(0)$ without any additional heating (thin lines in lower panels).

and LHCD $20 \mathrm{~s}$ earlier (Fig.2, right panel). It should be noted that it is not allowed to advance NBI because of the risk of shine through. As seen from fig. 3 also in this case a good hybrid $q$ profile is reached at the end of the ramp-up.

Regarding sensitivity of the results to the assumptions, following parameters were varied: $T_{\mathrm{e}, \mathrm{i}}$ (edge) (by 40\%), $n_{\mathrm{e}}$ (by 40\%), $n_{\mathrm{e}}$ profile shape (parabolic vs. flat) and $Z_{\text {eff }}$. We will only consider the scaling model $(\mathrm{H}=0.4)$ here; the sensitivity of the simulations to these changes when using the Bohm/gyro-Bohm model is quite similar and can be 


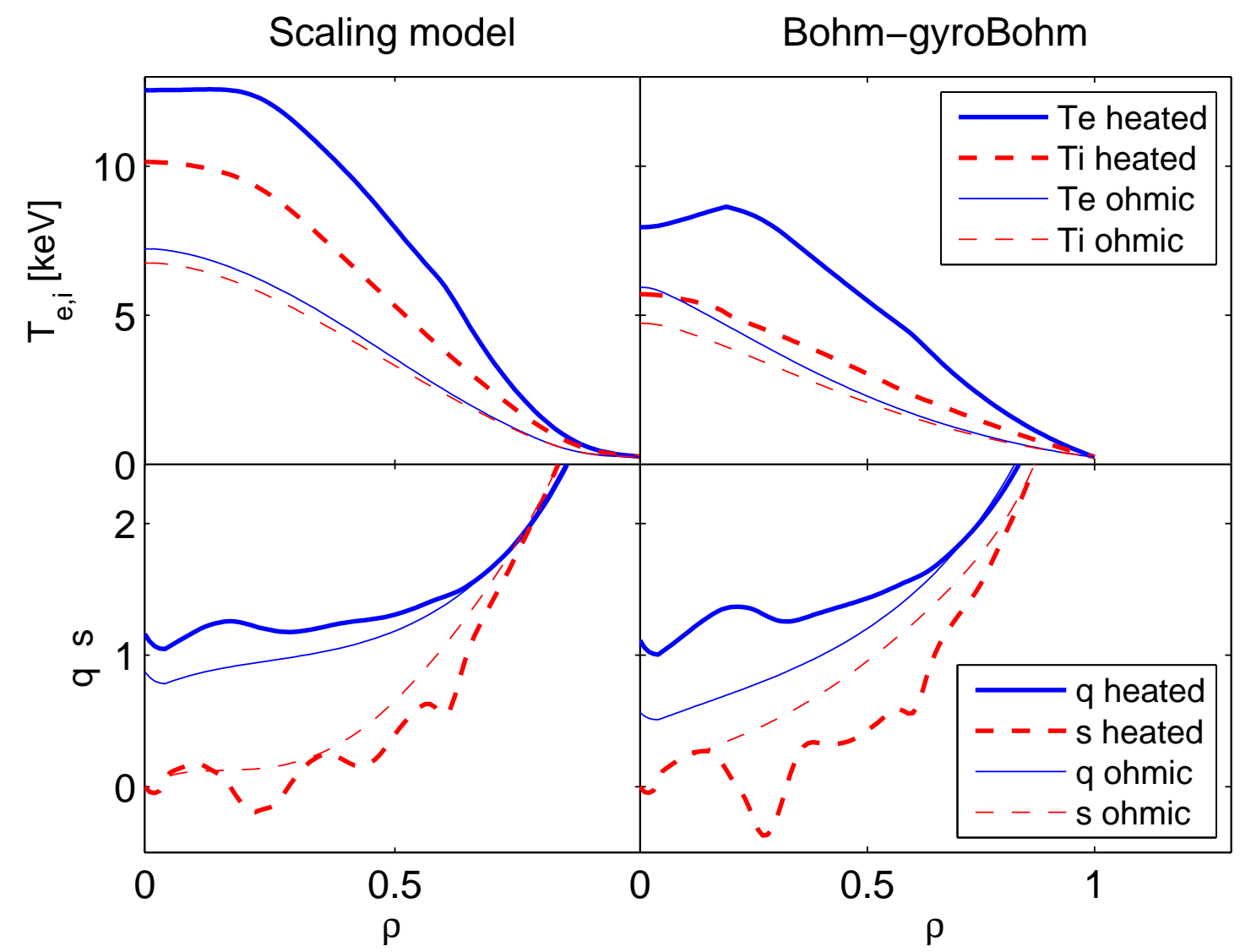

Figure 3. $T_{\mathrm{e}, \mathrm{i}} q$ and $s$ profiles for the same cases and with the same line coding as the previous figure.

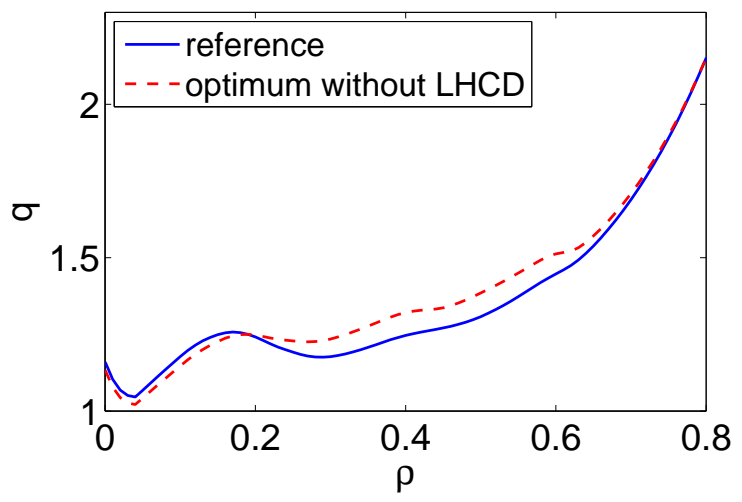

Figure 4. $q$ profiles at $80 \mathrm{~s}$ for the reference case (blue) and for a case where LHCD (3 $\mathrm{MW}$ ) was replaced by extra $8 \mathrm{MW}$ of ECCD (red dashed line).

accounted for in the same way.

(i) varying edge $T_{\mathrm{e}}$ gives only a modest change of $l_{\mathrm{i}}(\simeq 0.04)$ and a tiny change of $q$, so poses no problem.

(ii) $n_{\mathrm{e}}$ peaking: A flatter $n_{\mathrm{e}}$ profile would cause an increased peaking of $T_{\mathrm{e}}$, hence a 
faster current diffusion in the outer part of the plasma and a more peaked final current density profile; on the other hand the higher central $T_{\mathrm{e}}$ would slow down the current diffusion to the centre. Simulations show that the latter effect is less important, so the net effect is a more peaked current density profile: in an ITER ramp-up without additional heating, in a case with $n_{\mathrm{e}}(0) /\left\langle n_{\mathrm{e}}\right\rangle=1.05$ instead of 1.3 the moment that $q(0)$ reaches $1\left(t\left(q_{0}=1\right)\right)$ is shifted earlier by $\sim 10 \mathrm{~s}$. This can be compensated for by a corresponding earlier start of the additional heating. The opposite trend applies in case of a more peaked $n_{\mathrm{e}}$ profile and is accounted for in a similar way by delaying the heating, as shown in Fig.5.

(iii) $Z_{\text {eff }}$ : A $30 \%$ higher/lower value of $Z_{\text {eff }}$ causes a faster/slower current diffusion, and a shift of $t\left(q_{0}=1\right)$ of $\sim 10 \mathrm{~s}$, which can be compensated for like the previous case.

(iv) $n_{\mathrm{e}}$ : We only consider the effect of a $40 \%$ higher $n_{\mathrm{e}}$. Again this causes (due to lower $T_{\mathrm{e}}$ ) faster current diffusion. Since now also $P_{\text {LHthr }}$ is higher by $\simeq 10 \mathrm{MW}$, the applied power can be higher by this amount; moreover higher $n_{\mathrm{e}}$ allows earlier application of NBI. The thus adapted heating scheme, together with the time traces of $l_{\mathrm{i}}$ and $q(0)$, is shown in Fig.6. In this way the the flat $q$ profile is restored, see Fig.7.

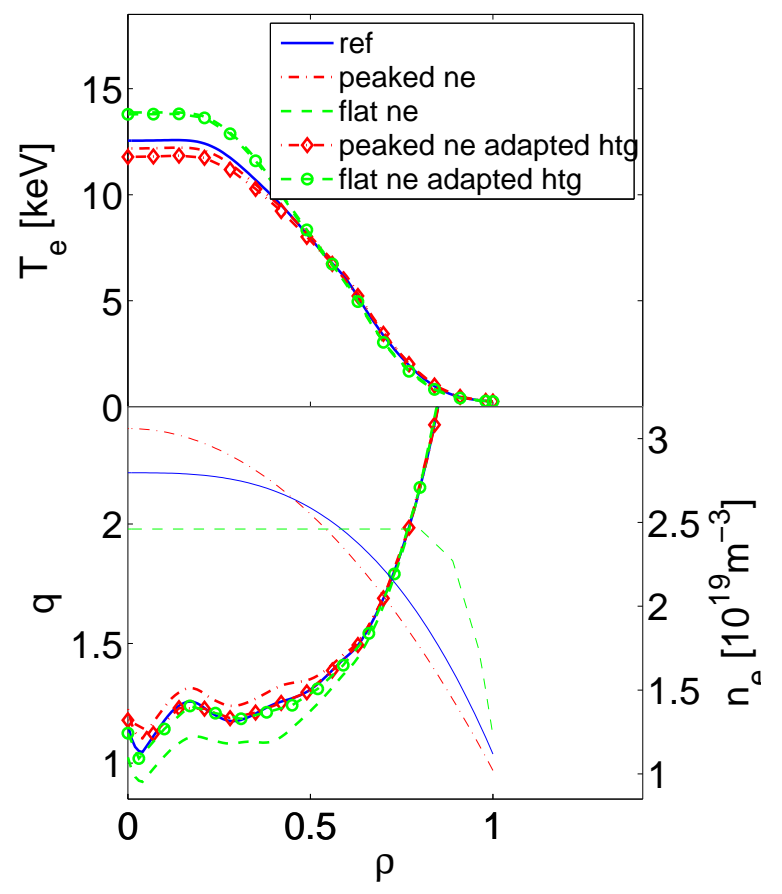

Figure 5. Effect of flat or extra peaked $n_{\mathrm{e}}$ profile. Shown are profiles of $T_{\mathrm{e}}$ (upper panel) and $q$ (lower panel) at $80 \mathrm{~s}$, without (dashed and dashed-dotted lines) and with adapted heating scheme (lines with symbols). In the lower panel also $n_{\mathrm{e}}$ is plotted (thin lines). The values of $n_{\mathrm{e}}(0) /\left\langle n_{\mathrm{e}}\right\rangle$ are $1.30,1.05$ and 1.47 , respectively, for the three cases shown. The heating was moved earlier/later by 10 and 6 s, respectively.

The figure of merit $(\mathcal{F})$, as defined in section 3 can be used to verify that the shifts in heating scheme, as indicated above, indeed restore the optimized $q$ profile. As an example, Fig. 8 shows the time traces of $\mathcal{F}$ for the reference case, and the cases with 
high $\mathrm{n}_{\mathrm{e}}$

high $\mathrm{n}_{\mathrm{e}}$ adapted heating

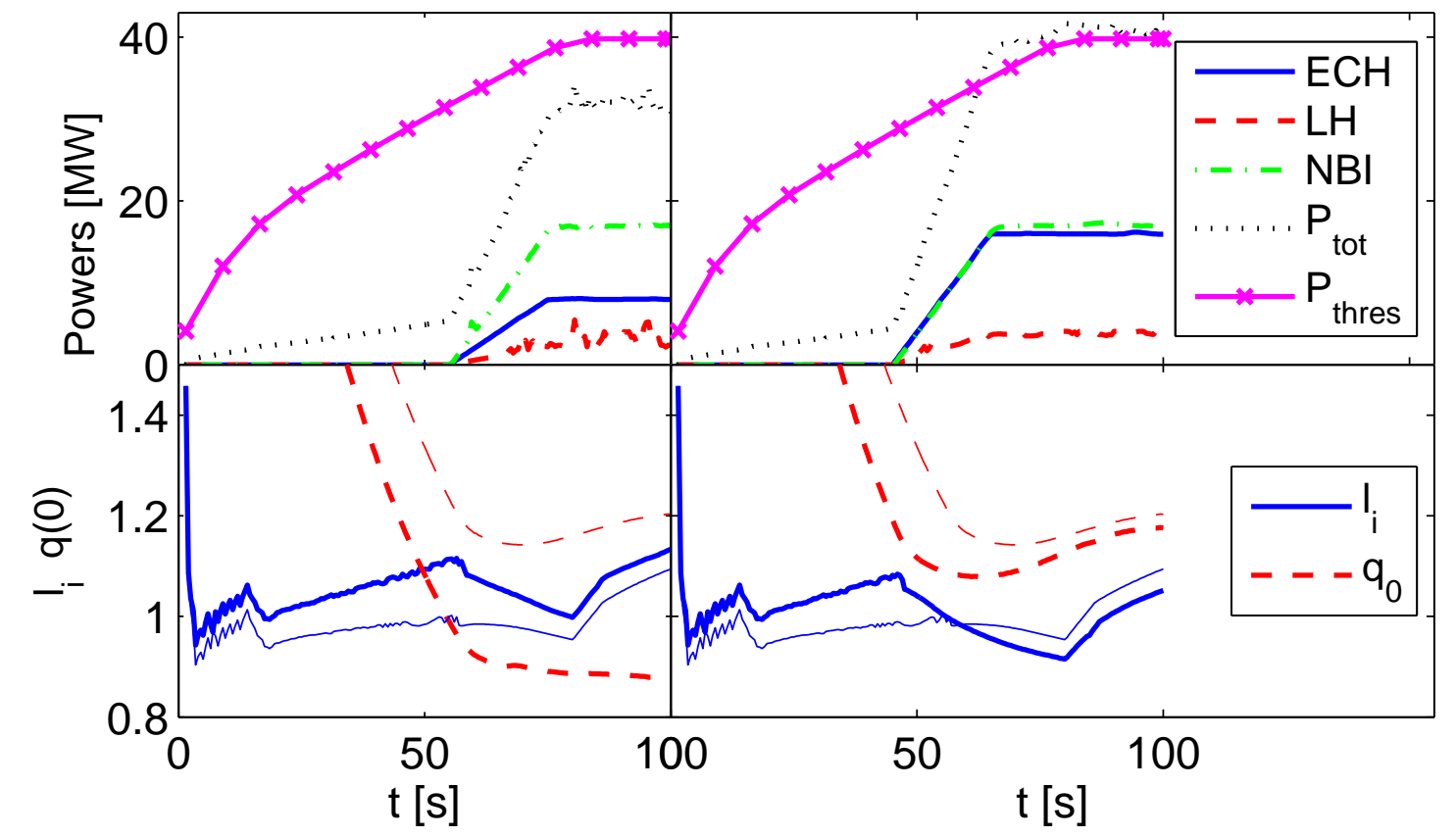

Figure 6. Time traces of powers, $q(0)$ and $l_{\mathrm{i}}$ for the high $n_{\mathrm{e}}$ case with the heating scheme of the reference scenario (left) and with adapted heating scheme (right). For comparison the lower panels also show the time traces of $q(0)$ and $l_{\mathrm{i}}$ of the reference scenario (thin lines). Without modified heating scheme, $q(0)$ drops far below 1 and $l_{\mathrm{i}}$ rises too high (i.e. above 1); these unwanted features are avoided with the adapted heating scheme. The LHCD power time traces are somewhat ragged due to the fact that the calculated absorbed power is plotted, which may be different from the prescribed power.

peaked $n_{\mathrm{e}}$ both without and with adapted heating. As can been seen, indeed the high value of $\mathcal{F}$ at the end of the current ramp-up at $80 \mathrm{~s}$ is restored with the adapted heating scheme.

The sensitivity of the current diffusion on parameters that are not easily controlled, shows that development of real time control is important to reach the target $q$ profile. As a first step in that direction, a real-time adaptive determination of auxiliary power switch-on time could be easily implemented on the basis of the present simulations, since for many parameters a deviation of the expected value can be accounted for by a simple shift of the heating wave form. Let $\mathcal{P}=\left\{\mathcal{P}_{i} \mid i=1, \ldots, N\right\}$ denote the values of a set of observables, like $Z_{\text {eff }},\left\langle n_{\mathrm{e}}\right\rangle$, etc. Let $\mathcal{P}^{0}=\left\{\mathcal{P}^{0}{ }_{i} \mid i=1, \ldots, N\right\}$ be the values of the reference case, and let $t^{0}$ be the starting time of the external heating in this case. Then the start time of the heating could depend on the measured values of the observables in the early phase of the current ramp-up as follows:

$$
t=t^{0}+\sum_{i=1, \ldots, N} \alpha_{i} \frac{\mathcal{P}_{i}-\mathcal{P}^{0}{ }_{i}}{\mathcal{P}^{0}{ }_{i}}
$$




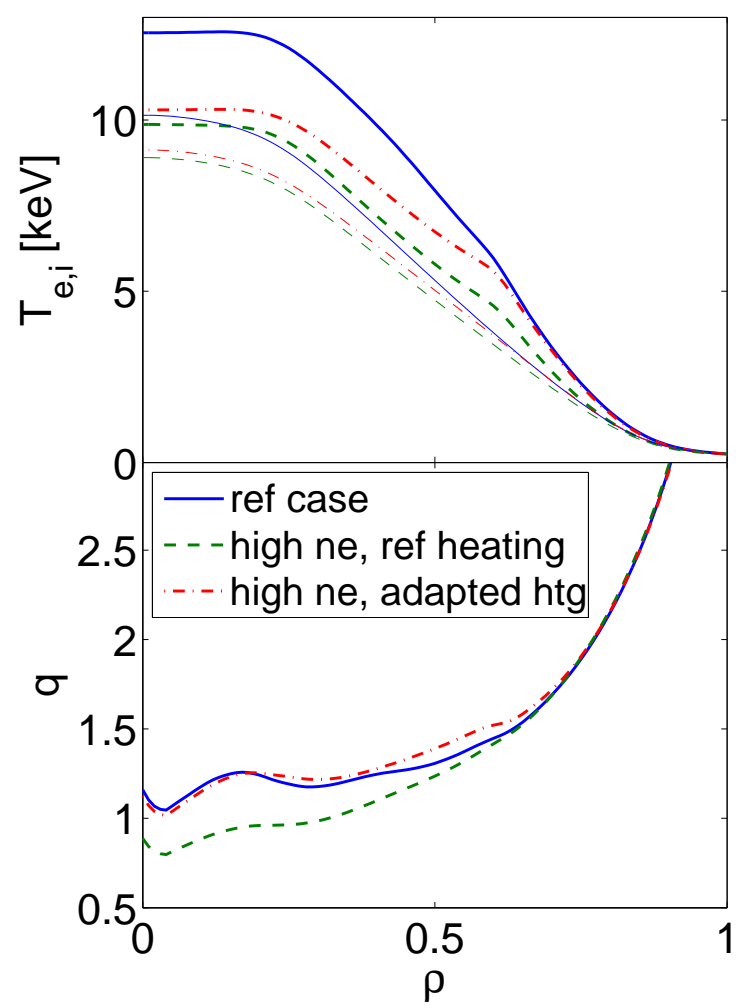

Figure 7. Profiles of $T_{\mathrm{e}}$ (upper panel, thick lines), $T_{\mathrm{i}}$ (upper panel, thin lines) and $q$ (lower panel) at $80 \mathrm{~s}$ for the same cases as Fig.6: reference case (full blue lines), high density case with unchanged heating (dashed green) and high density case with optimized heating scheme (dashed-dotted red).

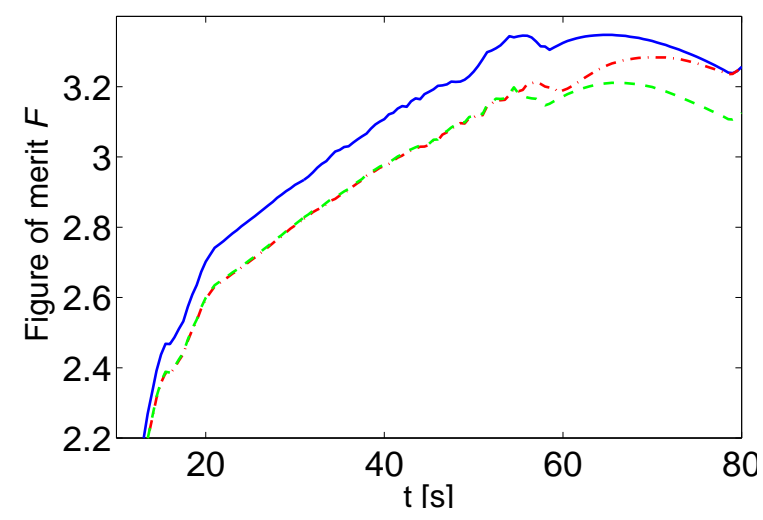

Figure 8. Time traces of $\mathcal{F}$ (see text) for the reference case (blue full line) and the cases with peaked $n_{\mathrm{e}}$ without and with adapted heating (green dashed and red dasheddotted line, respectively). Indeed the high value of $\mathcal{F}$ at the end of the ramp-up is restored with the adapted heating scheme.

where the values of the $\alpha_{i}$ can be determined from sensitivity analyses as presented earlier in this section. For example, let $\mathcal{P}_{1}$ be the value of $Z_{\text {eff }}$, then the sensitivity analysis showed that a time shift of $10 \mathrm{~s}$ was needed when the value deviated by $30 \%$ 
from the reference value, i.e. $\alpha_{1}=-10 / 0.3=-33 \mathrm{~s}$, with a minus sign because higher $Z_{\text {eff }}$ needs earlier heating. Similarly, of one takes for $\mathcal{P}_{2}$ the value of $n_{\mathrm{e}}(0) /\left\langle n_{\mathrm{e}}\right\rangle$, then the previous analysis showed that a peaking factor of 1.05 instead of 1.3 needed $10 \mathrm{~s}$ later heating, so $\alpha_{2} \cdot((1.05-1.3) / 1.3)=-10 \mathrm{~s}$, i.e. $\alpha_{2}=52 \mathrm{~s}$.

As an example, Eq.4 is applied to a case with flat $n_{\mathrm{e}}$ and $15 \%$ higher $Z_{\text {eff }}$, where Eq. 4 would prescribe a forward shift of the heating by 15 s. Figure 9 shows that in this way indeed an excellent reproduction of the optimum $q$ profile was obtained.

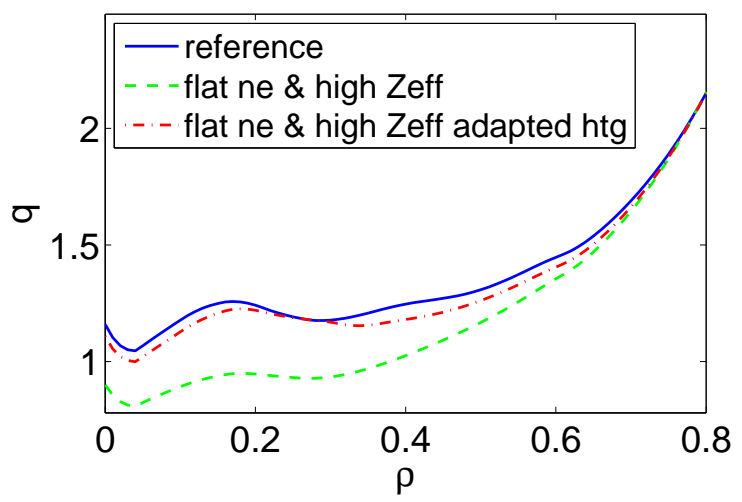

Figure 9. Effect of combination of flat $n_{\mathrm{e}}$ profile and higher $Z_{\mathrm{eff}}$ on $q$ profile at end of ramp-up (green dashed line), and result of applying modified heating scheme as prescribed by Eq.4 (red dashed-dotted line, see text).

Of course this simple approach has some limitations. First, a non-linear response may be needed when larger deviations from the expected values are encountered. More importantly, some plasma parameters which affect the $q$ profile evolution are not experimentally available during the early phase of the discharge, e.g. the $Z_{\text {eff }}$ profile shape. Nevertheless, the example given above shows that a simple real-time adaptive determination of auxiliary power switch-on time can already yield very useful results.

Alternative break down scenarios would of course also affect the $q$ profile evolution. As an example, one could envisage breakdown at the HFS instead of at the LFS, as is considered by the ITER team recently. The different geometry in the very early phase of the discharge leads to a modified current diffusion. However, the effect on the current density evolution turns out to be negligible after $\sim 50$ s, see Fig.10.

\section{Current ramp rate and current overshoot}

So far, all simulations were done with a moderate current ramp rate, reaching the flat top of $12 \mathrm{MA}$ at $80 \mathrm{~s}$. However, the ITER hardware would allow for a faster current rise. Moreover, recent JET experiments have shown that a current overshoot may lead to better hybrid characteristics, in terms of $q$ profile shape and confinement [36]. Also of relevance is whether flux consumption could be diminished in this way.

Therefore simulations have been done with a higher current ramp rate, reaching the flat top of $12 \mathrm{MA}$ at $60 \mathrm{~s}$, and these simulations have been further modified by adding a 

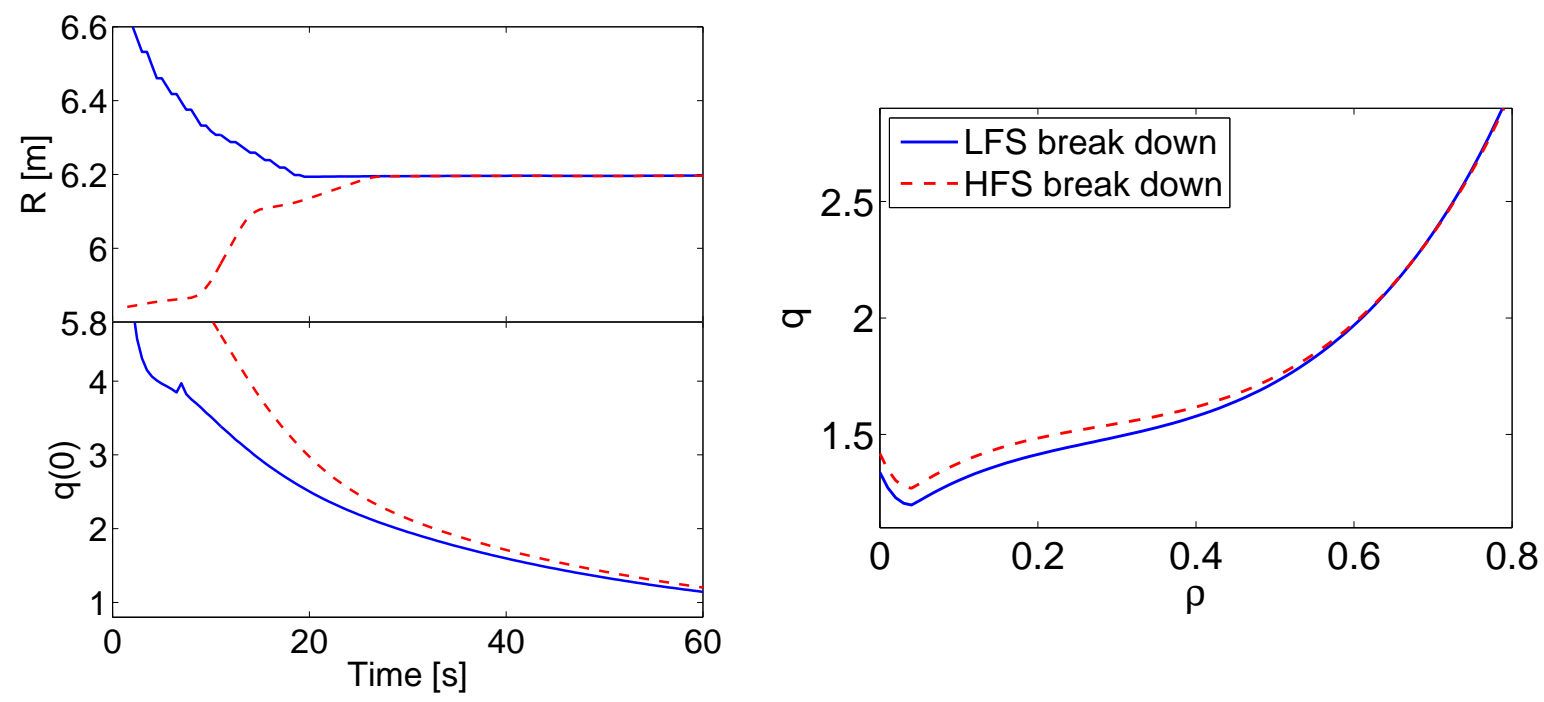

Figure 10. Left: time traces of $R_{0}$ and $q(0)$ for the normal breakdown at LFS (blue full line) and for alternative breakdown at HFS (red dotted line). Right: q profiles at 50 s for the same cases.

current overshoot up to $14 \mathrm{MA}$ at $70 \mathrm{~s}$, lasting for $10 \mathrm{~s}$. The start of the external heating has been shifted forward by $5 \mathrm{~s}$ for these two runs. The time evolution of $I_{\mathrm{p}}, P_{\text {total }}, l_{\mathrm{i}}$, $q(0)$, resistive flux consumption (to be discussed in next section), and $\mathcal{F}$ are plotted in Fig.11. Figure 12 shows the $q$ profiles at the start of the current flat-top for these two cases, i.e. at 60 and $90 \mathrm{~s}$, respectively; for comparison also the $q$ profile of the reference case is shown at the start of the current flat-top (80 s). Since part of the difference may be caused by the different duration of the ramp phases, also the three $q$ profiles are shown at $90 \mathrm{~s}$ (lines with diamonds/circles). In both comparisons the current overshoot shows by far the most favourable $q$ profile.

It should be noted that a control oriented approach, when applied to TCV, also showed the favourable effect of a current overshoot [37]. In this approach the tokamak actuator time evolution required to optimally reach a given point in the tokamak operating space while satisfying a set of constraints, is found by solving a non-linear, constrained, finite-time optimal control problem.

\section{Operational limits and flux consumption}

It is appropriate to come back now to the other two issues mentioned in the introduction: operational limits as posed by the central solenoid (CS) and poloidal field (PF) coils, and flux consumption.

By post processing the simulation results with the free boundary equilibrium code FREEBIE [16], run in Poynting mode, it has been checked that the simulations presented are within the boundaries put by the design values of the CS and PF coils. The most critical coils turn out to be the central solenoid coil CS1ULU+CS1ULL and the first 


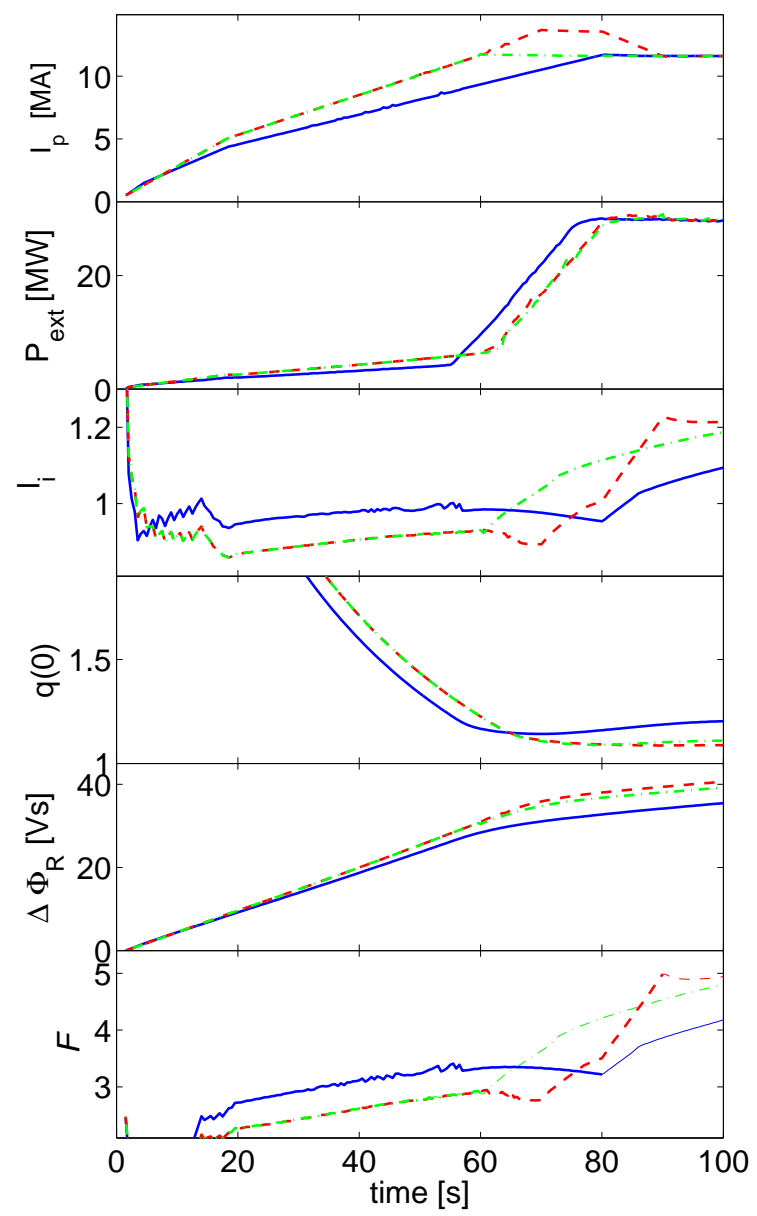

Figure 11. Comparison of the reference case (blue full lines) with optimized cases with faster current rise (green dashed-dotted lines) and with current overshoot (red dashed lines). Shown are, from top to bottom, time traces of $I_{\mathrm{p}}, P_{\mathrm{total}}, l_{\mathrm{i}}, q(0)$, resistive flux consumption, and $\mathcal{F}$.

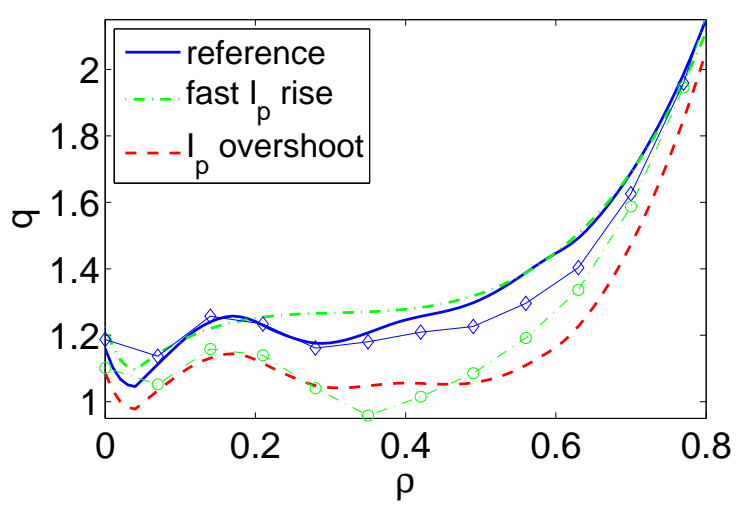

Figure 12. $q$ profiles at the start of the flat top, i.e. at 80, 60 and $90 \mathrm{~s}$, respectively, for the same 3 cases as the previous figure, with the same colour coding. For comparison all $q$ profiles are also shown at $90 \mathrm{~s}$ (lines with diamonds/circles. 
poloidal field coil PF1. Figure 13 shows the currents in these coils for three cases: the reference case without additional heating, a typical case with additional heating (similar to the reference case), and the case with fast current rise and current overshoot. All cases are within the limits. However, one could extrapolate that the current rise cannot be much faster without touching the limit of coil CS1ULU+CS1ULL. In order to allow
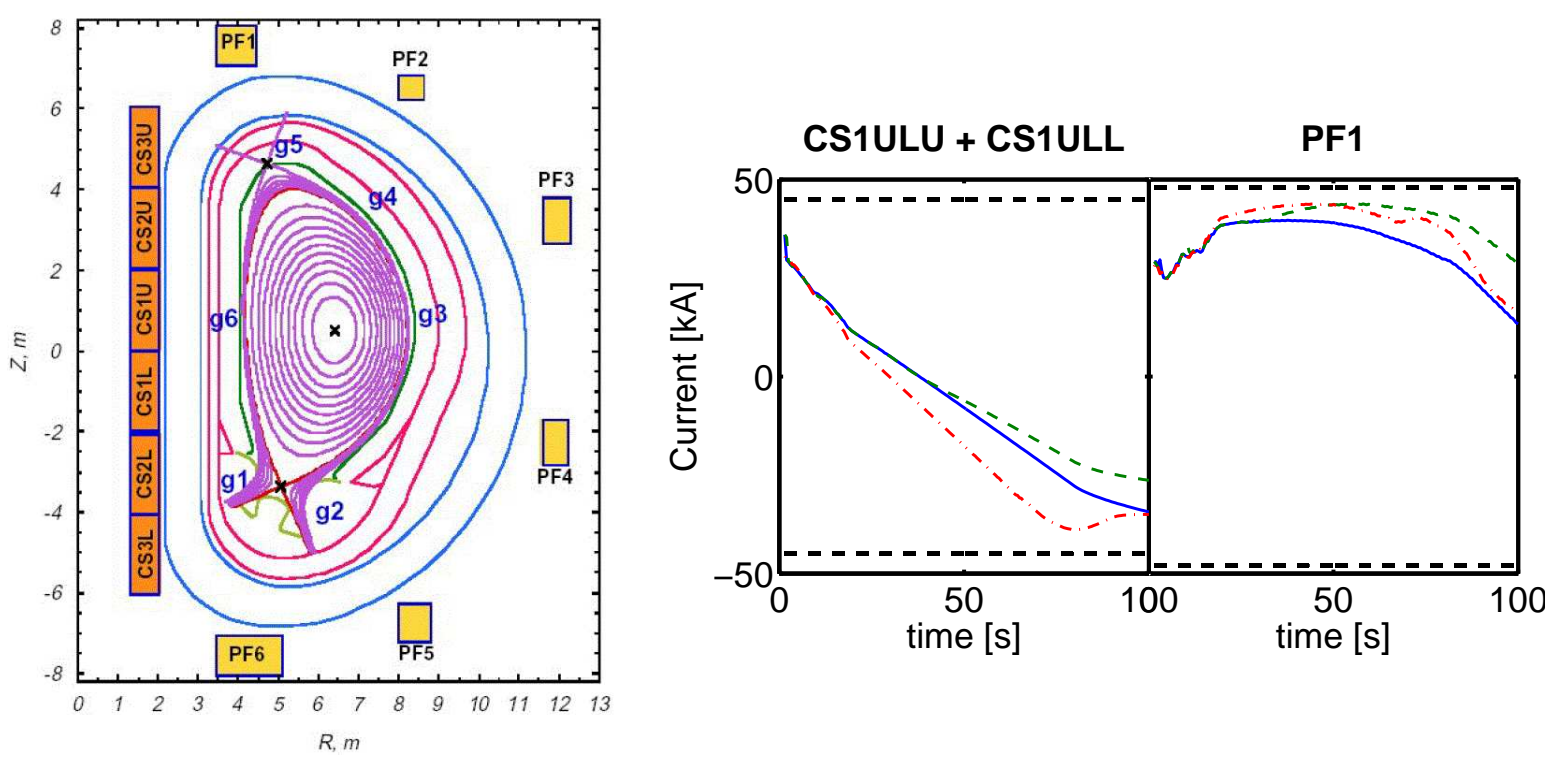

Figure 13. Lay-out of the ITER coil design (left), and some of the coil currents as calculated by FREEBIE (right). Shown are the currents in the two most critical coils: the central solenoid coils CS1ULU+CS1ULL, and the poloidal field coil PF1 for 3 cases: the reference case without additional heating (blue full line), a typical case with additional heating (green dashed), and the case with fast current rise and current overshoot as described in Section' (red dashed-dotted line). The maximum and minimum allowed currents are plotted in black.

for a long flat-top phase, it is desirable to reduce the flux consumption during the current rise phase as much as possible. For an analysis of the flux consumption, we distinguish between resistive and inductive flux $\left(\Delta \Phi_{R}\right.$ and $\left.\Delta \Phi_{I}\right)$. We follow the method as first described by Ejima et al [38], and later on applied to e.g. NSTX and DIII-D [39, 40], applying Poynting's theorem to the poloidal field. The total poloidal flux change at the plasma surface is expressed as

$$
\Delta \Psi_{\mathrm{S}}(t)=\Delta \Psi_{\mathrm{I}}(t)+\Delta \Psi_{\mathrm{R}}(t)
$$

with

$$
\begin{gathered}
\Delta \Psi_{\mathrm{I}}(t)=\int_{0}^{t} \frac{d t^{\prime}}{I_{\mathrm{p}}} \int_{V} \frac{\partial}{\partial t}\left(\frac{B_{\mathrm{pol}}^{2}}{2 \mu_{0}}\right) d V \\
\Delta \Psi_{\mathrm{R}}(t)=\int_{0}^{t} \frac{d t^{\prime}}{I_{\mathrm{p}}} \int_{V} j_{\phi} E_{\phi} d V
\end{gathered}
$$

where $B_{\mathrm{pol}}, j_{\phi}$ and $E_{\phi}$ denote the poloidal magnetic field and the toroidal current density and electric field, respectively. 
$\Delta \Psi_{\text {I }}$ at the end of the current ramp-up is determined by the poloidal field, e.g. the $q$ profile, attained; since this should always be close to the same, ideal shape, $\Delta \Psi_{\mathrm{I}}$ will always be more or less the same at the end of the ramp-up. Hence, if flux consumption is to be saved, it should be in the resistive part; therefore, we restrict the analysis to this part. It should be noted that at the end of the ramp-up phase both parts are of the same order of magnitude.

One could envisage two ways to reduce $\Delta \Psi_{\mathrm{R}}$ : by (very) early heating, and by early transition to the $\mathrm{H}$-mode. For both options one example which is believed to be representative has been chosen and has been simulated. The early heating example has, compared to the reference case, starting from $30 \mathrm{~s}$ additional $10 \mathrm{MW}$ of LHCD and $6 \mathrm{MW}$ of ECRH from the equatorial launcher; both induce considerable core heating and current drive. The example with early H-mode transition has full (33 MW) NBI power from $50 \mathrm{~s}$; the L-H transition is forced at $55 \mathrm{~s}$, with a pedestal of $4 \mathrm{keV}$ and high density ( $90 \%$ of $n_{\mathrm{e}}^{\mathrm{Gw}}$ ) after the transition. The H-mode phase was simulated both with the empirical scaling model (now assuming $H_{\mathrm{IPB} 98}=0.8$ ) and with the GLF23 transport model, with quite similar results. Figure 14 shows the total external heating power, $\left\langle T_{\mathrm{e}}\right\rangle$, the total non-inductive current (including bootstrap current), $l_{\mathrm{i}}$ and $\Delta \Psi_{\mathrm{R}}$ for these two examples (using scaling model for the H-mode phase), together with the reference case. Indeed the higher $T_{\mathrm{e}}$ and the increased non-inductive current lead to a significant reduction of the resistive flux consumption in both examples. Early heating turns out to be the most effective; it should be noted, however, that $l_{\mathrm{i}}$ rises slightly above 1 with early heating, which might pose operational problems.

The saving in flux consumption comes at a price: both examples feature a suboptimum $q$ profile at the end of the ramp-up, as shown in Fig.15. Moreover it should be noted that early heating leads to a rather large reversed shear region, which might be a good start for the steady state scenario but not for the hybrid scenario.

It should be noted that a similar dependence between flux consumption and $q$ profile shape was seen in the simulations with faster current ramp-up and current overshoot: there the improved $q$ profile came at the price of an enhanced resistive flux consumption $\left(\Delta \Psi_{\mathrm{R}}\right.$, see $4^{\text {th }}$ panel of Fig.11). Therefore a trade-off has to be made between $\Delta \Psi_{\mathrm{R}}$ and optimization of the $q$ profile.

A useful figure of merit for the resistive flux consumption during the current rampup is the so-called Ejima coefficient, defined as $C_{\mathrm{E}}=\Delta \Psi_{\mathrm{R}} /\left(\mu_{0} R_{0} I_{\mathrm{p}}\right)$, calculated at the end of the current ramp-up [38]. Figure 16 shows the two parameters to judge this trade-off, viz. $C_{\mathrm{E}}$ and $\mathcal{F}$ both for the two examples with reduced flux consumption as discussed above, and for the two cases with fast current ramp and current overshoot of the previous section, together with the reference case. The total input energy during the ramp-up is used as horizontal axis.

A reduction of $C_{\mathrm{E}}$ by 0.05 corresponds to a saving of $\simeq 4.7 \mathrm{Vs}$. With an expected $V_{\text {loop }}$ during the flat-top of the hybrid scenario of $0.02-0.05 \mathrm{~V}$ (mainly depending on assumption of pedestal height, [3]), this would correspond to an extension of the flat top duration by 100-150 s. This clearly shows the significance of the trade-off between 


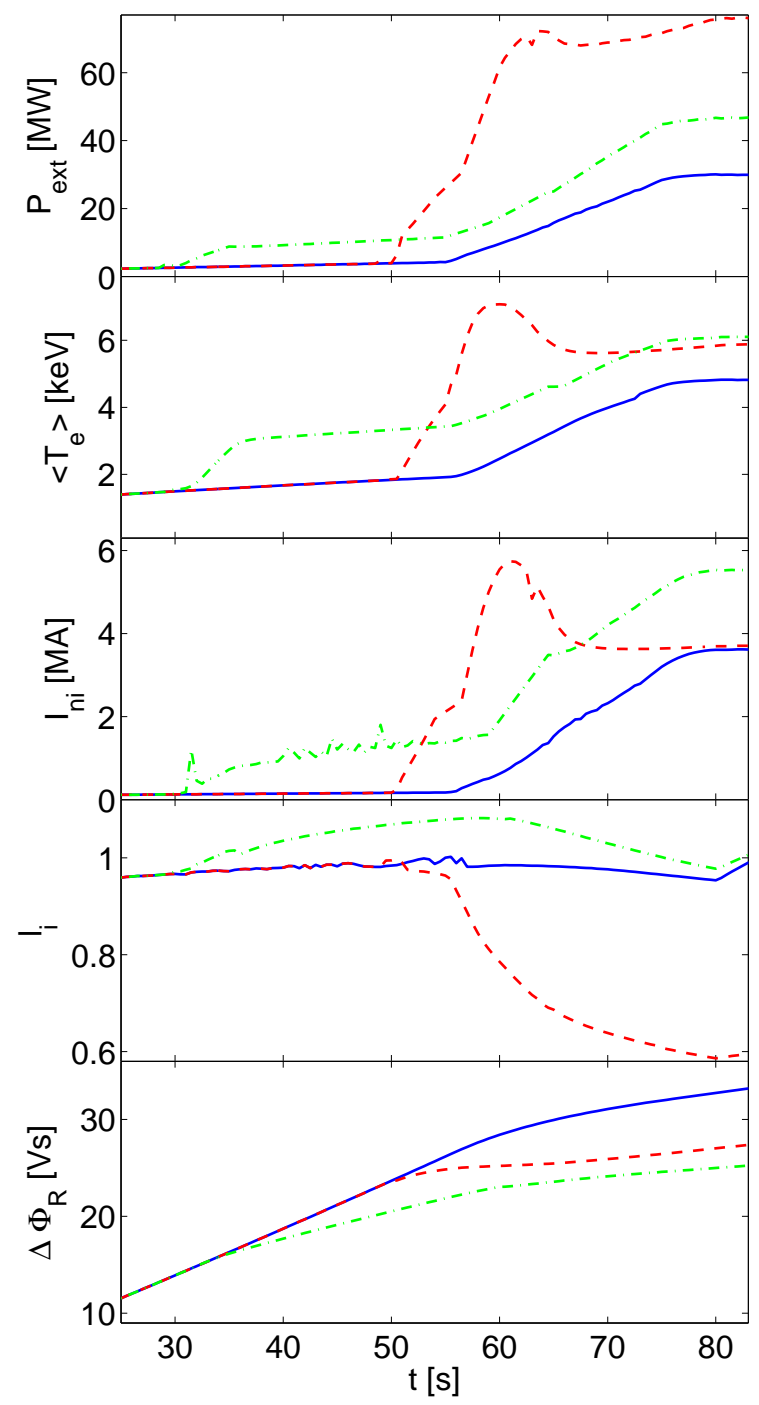

Figure 14. Total external heating power, $\left\langle T_{\mathrm{e}}\right\rangle$, total non-inductive current (including bootstrap current), $l_{\mathrm{i}}$ and $\Delta \Psi_{\mathrm{R}}$ for the reference case (blue full line), an example with additional early heating (green dashed-dotted) and an example of early H-mode transition (red dashed line).

flux consumption and optimization of the $q$ profile: the flat-top can be extended by some 200-250 s by early heating or early H-mode, i.e. 10\% for an expected flat top duration of $\sim 3000 \mathrm{~s}$, however at the cost of a strong reversed shear region or a less well-developed $q$ profile, respectively. On the other hand, fast current rise combined with current overshoot leads to a superior $q$ profile, but at the cost of a reduced flat-top by some $200-300$ s, i.e. $\sim 10 \%$. In practice this reduction might be much smaller, since an optimized $q$ profile leads to better plasma performance and thus lower $V_{\text {loop }}$ during the flat-top. Moreover, one should keep in mind that one of the goals of the hybrid scenario is to maximize the neutron fluence (total number of neutrons per shot). Since $P_{\text {fus }}$ is approximately proportional to $\mathcal{F}[3]$, it may well be that the neutron fluence is 


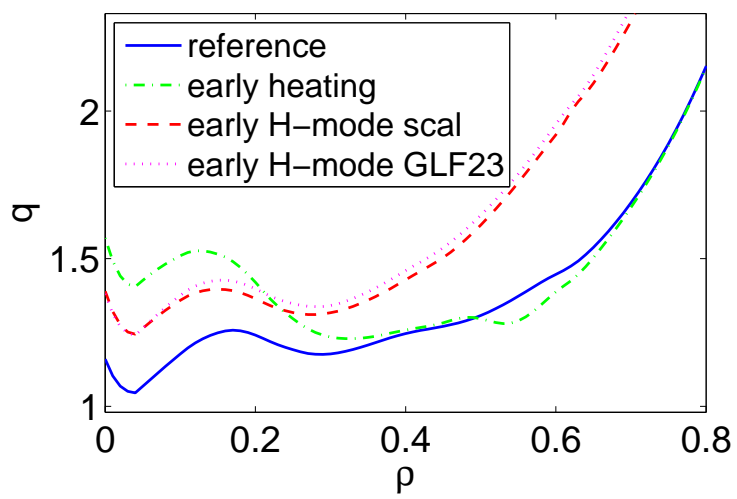

Figure 15. $q$ profiles at $80 \mathrm{~s}$ for the reference case (blue full line), with early heating (green dashed-dotted) and with transition to H-mode after 55s, using 2 different transport models for the H-mode phase (red dashed/magenta dotted for scaling model/GLF23, respectively).

higher for the case with current overshoot.

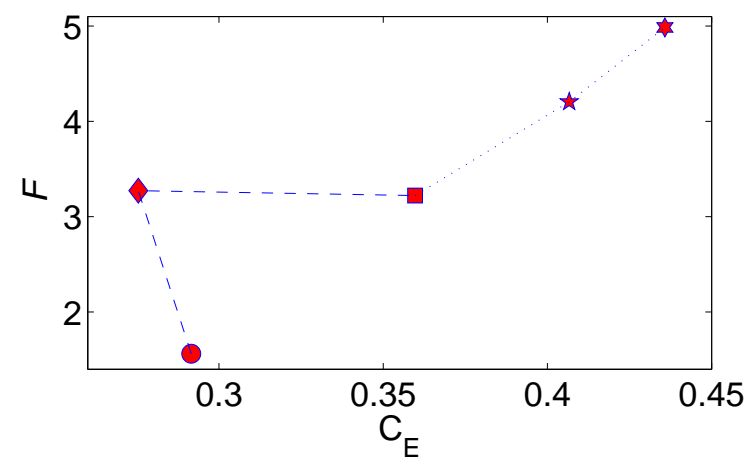

Figure 16. Figure of merit $(\mathcal{F})$ plotted vs. Ejima coefficient $\left(C_{\mathrm{E}}\right)$ at the end of the ramp-up phase for the reference case (square), the examples with early heating (diamond) and with transition to H-mode after $55 \mathrm{~s}$ (circle), and for the optimized cases with fast current ramp (pentagram) and current overshoot (hexagram).

\section{Extrapolation to burn phase}

Since the final goal is to sustain the optimized $q$ profile during the $\sim 3000$ s flat top, in order to maximize the neutron fluence, two more questions are important: how does the $q$ profile react to the L-H transition, and can $q$ be held stationary during the long flat top.

Regarding the first question: based on the reference case, preliminary simulations were done to assess the evolution of the q profile during the $\mathrm{L}-\mathrm{H}$ transition. To this end, in a time window of $20 \mathrm{~s}$ immediately after the end of the current ramp-up, the external power was raised to $70 \mathrm{MW}(33 \mathrm{MW} \mathrm{NBI}+37 \mathrm{MW}$ ECRH), i.e. clearly above the L-H 
threshold, and in the modelling the $\mathrm{L}-\mathrm{H}$ transition was forced by imposing a pedestal of $4 \mathrm{keV}$. At the same time the density was raised to the target density for the hybrid scenario $\left(\sim 9 \cdot 10^{19} \mathrm{~m}^{-3}\right) ; \alpha$ particle heating was taken into account in the modelling. It turned out that during this transition the core $q$ profile was preserved very well.

The second question has already been addressed in earlier work, which showed that, under reasonable assumptions for the pedestal, indeed the $q$ can be held stationary during the long flat top [3].

It should be noted that, in case of a non-optimized $q$ profile at the start of the burn phase, it is still possible to optimize the $q$ profile during the burn phase itself. However, due to the very low resistivity in this phase, this may take up to $\sim 1000 \mathrm{~s}$. Therefore, with a non-optimized $q$ profile at the start of the burn phase, one might expect a significantly reduced fusion power during the first $\sim 1000 \mathrm{~s}$ of the total burn phase of $\sim 3000 \mathrm{~s}$.

\section{Conclusions and Outlook}

The simulations presented in this paper show that the heating systems available at ITER allow, within the operational limits, the attainment of a hybrid $q$ profile at the end of the current ramp-up. A reference ramp-up scenario is reached by a combination of NBI, ECCD (UPL) and LHCD.

Since LHCD is not contained in the ITER baseline design, also simulations without LHCD have been performed. It turns out that the target q profile can also be achieved with a heating scheme using only NBI and ECCD. However, being the most efficient current driver, LHCD can play a crucial role in reducing the flux consumption during the ramp-up phase, and hence in extending the duration of the burn phase by $\sim 10 \%$.

The optimum heating scheme depends on the chosen transport model. Moreover, modified assumptions on $n_{\mathrm{e}}$ peaking, edge $T_{\mathrm{e}, \mathrm{i}}$ and $Z_{\mathrm{eff}}$ can be easily accounted for by a shift in start time of the heating scheme. A higher density during the ramp-up phase can be accounted for equally well, and might even be profitable because it gives more freedom in the application of the heat sources, while keeping the plasma $n$ L-mode.

The sensitivity of the current diffusion on parameters that are not easily controlled, shows that development of real time control is important to reach the target $q$ profile. As a first step in that direction, a real-time adaptive determination of auxiliary power switch-on time could be easily implemented on the basis of the simulations presented in this paper. A basic example of this was given in this paper.

Minimizing resistive flux consumption and optimizing the $q$ profile turn out to be conflicting requirements. A trade-off between these two requirements has to be made. In this paper it was shown that fast current ramp with current overshoot is at the one extreme, i.e. optimum $q$ profile at the cost of increased resistive flux consumption, whereas early $\mathrm{H}$-mode transition is at the other extreme.

The fact that current overshoot is useful in tailoring the $q$ profile was experimentally investigated at JET [36] and corroborated by theoretical work at TCV [37]. It has now 
for the first time proven to be a useful tool for ITER as well.

\section{References}

[1] Kessel C.E. et al 2009 Nucl. Fusion 49085034

[2] Gormezano C. et al Progress in the ITER Physics Basis Chapter 6: Steady State Operation 2007 Nucl. Fusion 47 S285

[3] Citrin J. et al 2010 Nucl. Fusion 50115007

[4] Jenkins I. et al 2010 Test of current ramp modelling for AT regimes in JET Proc. 37th EPS Conf. on Plasma Physics (Dublin, Ireland, 2010) P2.178 http://ocs.ciemat.es/EPS2010PAP/pdf/P2.178.pdf

[5] Citrin J. et al 2012 Plasma Phys. Control. Fusion 54065008

[6] Voitsekhovitch I. et al 2010 Plasma Phys. Control. Fusion 52105011

[7] Garcia J. et al 2011 Nucl. Fusion 51073019

[8] Hogeweij G.M.D. et al 2010 Current ramp-up in tokamaks: from present experiments to ITER scenarios Proc. 37th EPS Conf. on Plasma Physics (Dublin, Ireland, 2010) P1.1041 http://ocs.ciemat.es/EPS2010PAP/pdf/P1.1041.pdf

[9] Imbeaux F. et al 2011 Nucl. Fusion 51083026

[10] Erba M. et al Nucl. Fusion 1998381013

[11] Jardin S.C. et al Nucl. Fusion 199333371

[12] Casper T.A. et al 2011 Nucl. Fusion 51013001

[13] Artaud J.-F. et al 2010 Nucl. Fusion 50043001

[14] Huysmans G.T.A. et al 1991 CP90 Conf. on Comp. Physics (Singapore: Word Scientific) p 371

[15] Houlberg W.A. et al 1997 Phys. Plasmas 43230

[16] Kim S.H. et al 2012 A new free-boundary equilibrium evolution code, FREEBIE Proc. 39th EPS Conf. on Plasma Physics (Stockholm, Sweden, 2012) P4.023 http://ocs.ciemat.es/epsicpp2012pap/pdf/P4.023.pdf

[17] Schneider M. et al 2009 Nucl. Fusion 49125005

[18] Schneider M. et al 2011 Nucl. Fusion 51063019

[19] Krivenski V. et al 1985 Nucl. Fusion 25127

[20] Peysson Y. et al 2008 EURATOM-CEA Report EUR-CEA-FC-1738

[21] Peysson Y. et al 1993 Plasma Phys. Control. Fusion 35 B253

[22] Peysson Y. et al 2011 Plasma Phys. Control. Fusion 53124028

[23] S.M. Kaye S.M. et al 1997 Nucl. Fusion 371303

[24] ITER Physics Expert Group on Confinement and Transport et al ITER Physics Basis, Chapter 2: Plasma confinement and transport 1999 Nucl. Fusion 392175

[25] Kardaun O. et al 2008 Nucl. Fusion 48099801

[26] Lukash V. et al 2007 Plasma Devices and Oper. 15283

[27] Weisen H. et al 2005 Nucl. Fusion 45 L1

[28] Martin Y.R. et al 2008 J.Phys.: Conf.Ser. 123012033

[29] Romanelli F. et al 1989 Phys. Fluids B 11018

[30] Connor J.W. et al 1994 Plasma Phys. Control. Fusion 36719

[31] Mantica P. et al 2011 Phys. Rev. Lett. 107135004

[32] Jenko F. et al 2001 Phys. Plasmas 84096

[33] Guo S.C. et al 1993 Phys. Fluids B 5520

[34] ITER Physics Expert Group on Energetic Particles et al ITER Physics Basis, Chapter 6: Plasma auxiliary heating and current drive 1999 Nucl. Fusion 392495

[35] Kim S.H. et al 2009 Plasma Phys. Control. Fusion 51065020

[36] Romanelli F. et al 2009 Nucl. Fusion 49104006

[37] Felici F. et al 2012 Plasma Phys. Control. Fusion 54025002

[38] Ejima S. 1982 Nucl. Fusion 221313 
[39] Menard J.E. et al 2001 Nucl. Fusion 411197

[40] Jackson G.L. et al 2009 Nucl. Fusion 49115027

\section{Acknowledgements}

This work, supported by the European Communities under the contract of Association between EURATOM/FOM, was carried out within the framework of the European Fusion Programme with financial support from NWO. The views and opinions expressed herein do not necessarily reflect those of the European Commission. This work is supported by NWO-RFBR Centre-of-Excellence on Fusion Physics and Technology (Grant nr. 047.018.002). 